\title{
Pattern Formation in Polymer Blend Thin Films: Surface Roughening Couples to Phase Separation
}

\author{
Sam Coveney and Nigel Clarke \\ Department of Physics and Astronomy, University of Sheffield, Hicks Building, Hounsfield Road, \\ Sheffield S3 7RH, United Kingdom
}

(Received 23 May 2014; revised manuscript received 4 August 2014; published 19 November 2014)

\begin{abstract}
We introduce a model for thin films of multicomponent fluids that includes lateral and vertical phase separation, preferential component attraction at both surfaces, and surface roughening. We apply our model to thin films of binary polymer blends, and use simulations of different surface-blend interaction regimes to investigate pattern formation. We demonstrate that surface roughening couples to phase separation. For films undergoing lateral phase separation via a transient wetting layer, this results in distinct stages of roughening as the film evolves between different phase equilibria.
\end{abstract}

Semiconducting polymer devices, such as photovoltaic films, can gain performance enhancements from phase separated morphologies. Since polymer films are prone to surface roughening, understanding how phase separation couples to dewetting is particularly important. This interplay was highlighted by Walheim et al. for spin-cast polymer films [1] and has been repeatedly observed, e.g., phase separation in symmetrically surface-segregating films [2-5], lateral phase separation via a transient wetting layer [6,7], and phase separation proceeding from the surface as the solvent evaporates [8]. Pattern formation in polymer blend thin films, in which surface roughening shadows the phase separated morphology, is incredibly common $[9,10]$. Theoretical results suggest that, generally, the coupling of phase separation and height variation makes films less stable [11] and can trigger instabilities [12,13].

A variety of models for multicomponent deformable fluid films have been investigated. The "Clarke model" (name introduced here) utilized nonequilibrium thermodynamics based upon a free energy functional, demonstrating that phase separation generally couples to dewetting [12]. A model based on the Navier-Stokes Cahn-Hilliard equations in the lubrication approximation showed that concentration gradients can create a roughened pattern that mirrors the underlying phase separation [14]. However, the film composition has no vertical dependence in these models, so a meaningful preferential surface attraction of blend components cannot be included. Two-layer models exist, including models with immiscible fluid layers $[15,16]$ and layers with a diffuse boundary for films with no preferential surface attraction [17]. However, a general vertical composition dependence, which could allow vertical phase separation to occur during a simulation, is typically not included [9], though such a case has been studied with regards to stability but not simulated [18]. An exception is a model of surface roughening of polymer blend films, although this model is not based upon a dewetting film [19].

This Letter presents a model formulation of a binary blend thin film which, by way of including a general vertical dependence of composition, can be utilized for a full time simulation of coupled dewetting and phase separation, including (i) both lateral and vertical phase separation, (ii) preferential attraction of the blend by both surfaces, and (iii) film height evolution. We show that our model can reproduce pattern formations observed in phase separating polymer films, and we explain how the stages of surface roughening correspond to stages of phase separation, which are determined by how the film evolves into, and between, different phase equilibria.

The central part of the Clarke model is a free energy functional depending on material volume fraction $\phi(y)$ and film height $h(y)$, given by $\mathcal{F}[\phi(y), h(y)]=\int f(\phi, h)+$ $h g(\phi) d y$ where $f(\phi, h)$ is the surface energy, $g(\phi)$ is the bulk free energy, and $y$ is the lateral direction parallel to the film substrate [12]. We first introduce a vertical dependence $z$, which for convenience we a priori discretize, replacing the coordinate $z$ with index $i$ :

$$
\mathcal{F}[\phi(i, y), h(y)]=\int f(\phi, h)+\sum_{i=1}^{D} \Delta z(y) g(\phi) d y,
$$

so that $h(y)=D \Delta z(y)$; i.e., the height at point $y$ is divided into $D$ grid cells of equal height $[\Delta z(y)$ does not depend on $i]$. We can write $\mathcal{F}=F^{\phi}+F^{h}+F^{\phi, h}$

$$
\begin{gathered}
F^{\phi}=\int f^{\phi}(\phi)+\sum_{i=1}^{D} \Delta z(y) g(\phi) d y, \\
F^{h}=\int f^{h}(h) d y, \quad F^{\phi, h}=\int f^{\phi, h}(\phi, h) d y,
\end{gathered}
$$


where we have separated the surface energy into $f(\phi, h)=f^{\phi}(\phi)+f^{h}(h)+f^{\phi, h}(\phi, h)$, where $f^{h}(h)$ and $f^{\phi}(\phi)$ depend only on $h$ and $\phi$, respectively, and $f^{\phi}(\phi, h)$ contains any cross terms. Figure 1 is a schematic of our model, explaining the average volume fraction $\bar{\phi}(y)=$ $D^{-1} \sum_{i=1}^{D} \phi(i, y)$ at point $y$ and the volume fraction $\phi(i, y)$ in individual grid cells.

Our dynamic model proceeds via two steps: a height evolution step and a diffusion step. For the height evolution, which involves lateral movement of material, we use the following coupled equations [12]:

$$
\begin{gathered}
\frac{\partial h(y)}{\partial t}=\nabla_{y} \cdot\left(\frac{h^{3}}{3 \eta} \nabla^{*} \mu_{c}\right), \\
\frac{\partial \bar{\phi}(y) h(y)}{\partial t}=\nabla_{y} \cdot\left(\frac{h^{3} \bar{\phi}}{3 \eta} \nabla^{*} \mu_{c}\right),
\end{gathered}
$$

and for diffusion with a nonuniform grid we use

$$
\frac{\partial \phi(i, y) \Delta z(y)}{\partial t}=M \nabla \cdot\left[\Delta z(y) \nabla \mu_{\phi \Delta z}(i, y)\right],
$$

where $M$ is the mobility. We have defined

$$
\begin{gathered}
\nabla^{*} \mu_{c}(y) \equiv \nabla_{y} \mu_{h}(y)+\bar{\phi} \frac{1}{D} \sum_{i=1}^{D} \nabla_{y} \mu_{\phi \Delta z}(i, y), \\
\mu_{h}(y) \equiv \frac{\delta F^{h}}{\delta h}+\frac{\delta F^{\phi, h}}{\delta h}+\frac{1}{D} \frac{\delta F^{\phi}}{\delta \Delta z}, \\
\mu_{\phi \Delta z}(i, y) \equiv \frac{\delta F^{\phi}}{\delta\left(\phi_{i} \Delta z\right)}+\frac{\delta F^{\phi, h}}{\delta\left(\phi_{i} \Delta z\right)} .
\end{gathered}
$$

Note the presence of functional derivatives. Our model satisfies the thermodynamic stability criterion required for an initially homogeneous film [11,12]. Model implementation is discussed in the Supplemental Material [20].

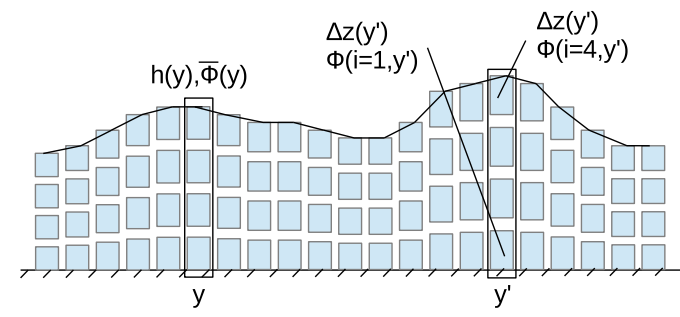

FIG. 1 (color online). 2D schematic of our model, representing a fluid layer with a deformable upper surface. Here, the vertical direction is divided into $D=4$ grid cells with height $\Delta z\left(y^{\prime}\right)=h\left(y^{\prime}\right) / D$, such that the cell height at $y^{\prime}$ is constant. Each cell has volume fraction $\phi\left(i, y^{\prime}\right)$, where index $i$ runs from 1 to $D$. The average volume fraction $\bar{\phi}(y)$ is an average over $i$ at point $y$.
To illustrate the model, we apply it to a symmetric binary polymer blend (components $A$ and $B$ ) between selectively attracting walls. The bulk free energy is given by $g(\phi)=$ $f_{F H}(\phi)+\kappa(\phi)(\nabla \phi)^{2}$ where $f_{F H}(\phi)=(1 / N)[\phi \ln (\phi)+$ $(1-\phi) \ln (1-\phi)]+\chi \phi(1-\phi)$ and $\kappa(\phi)=a^{2} / \phi(1-\phi)$, where $a$ is the underlying Flory-Huggins lattice spacing, $N$ is the degree of polymerization, and $\chi$ is the Flory-Huggins interaction parameter [23]. The $\phi$-dependant surface energy is given by $f^{\phi}(\phi)=f_{D}(\phi) \delta_{i D}+f_{1}(\phi) \delta_{i 1}$ where $f_{S}=$ $h_{S} \phi+(1 / 2) g_{S} \phi^{2}$ and $h_{S}$ and $g_{S}$ are phenomenological parameters [24,25]. $S=1, D$ and $\delta_{i S}$ is the Kronecker delta function, so the surface energies only act in the cells adjacent to the film surfaces. For the height-dependent surface energy we use $f^{h}(h)=\sigma\left[\nabla_{y} h(y)\right]^{2}+\epsilon / h^{8}$, which implies we have chosen the Hammaker constant to be zero. $f^{\phi, h}(\phi, h)=0$ for simplicity. In Eqs. (7)-(9) we must write $\phi \Delta z / \Delta z$ in place of $\phi$ when calculating functional derivatives [26].

We use the following parameters: $N=100, \bar{\phi}=0.5$ (unless specified), $a=1, \eta=1000, M=1, \epsilon=0.010$, $\sigma=0.01$ (unless specified). We perform 3D simulations with lateral dimensions $x$ and $y$. We scale space by $z^{\prime}=$ $\left|\chi-\chi_{S}\right|^{1 / 2} z / a$ (similarly for $x$ and $y$ ) and time by $\tau=N M\left|\chi-\chi_{S}\right|^{2} t / a^{2}$, where $\chi_{S}$ is the value of $\chi$ on the spinodal (for scaling, see Ref. [27]). We use a square simulation grid with $\Delta x^{\prime}=\Delta y^{\prime}=0.173$, and use $D=16$ grid cells to discretize the vertical direction. We used $\chi=0.050$ (although both $a$ and $\chi$ are both absorbed into the scaling). We mostly specialize to a symmetric polymer blend $\bar{\phi}(y)=0.5$ (except in Fig. 6) to focus on surface energy regimes (quoted in unscaled values): for the "antisymmetric" film $h_{1}=-0.05, g_{1}=0.18, h_{D}=-0.13$, $g_{D}=0.18$ of Figs. $2-3$ ( $B$-attracting substrate, $A$-attracting surface); for the asymmetric film of Figs. $4-5 h_{1}=-0.05$,
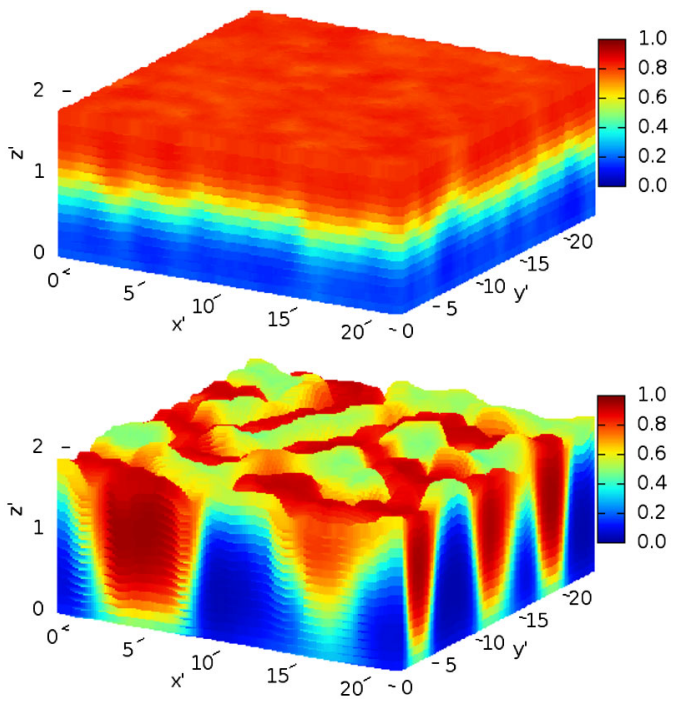

FIG. 2 (color online). Bilayer (top) at $\tau=100$ and laterally segregated state (bottom) at $\tau=1263$ for an antisymmetric film of average height $h^{\prime}=1.73$. 


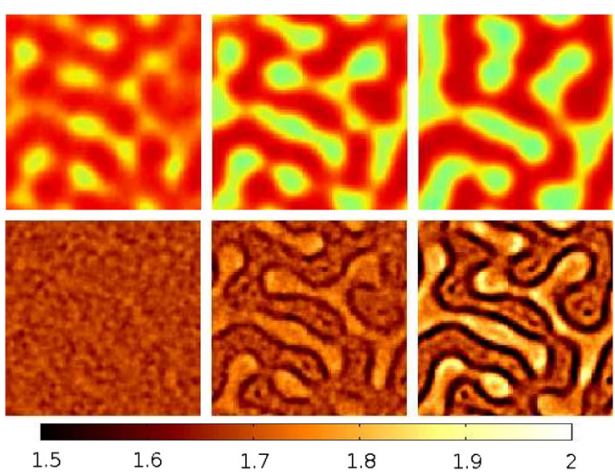

FIG. 3 (color online). Surface view for film shown in Fig. 2 (top: volume fraction, color bar of Fig. 2; bottom: film height, color bar shown). Left: breakup of the bilayer $(\tau=500)$; center: appearance of lateral phases $(\tau=830)$; right: further roughening as lateral phases coarsen $(\tau=1263)$. The surface morphology is bicontinuous, with the $B$-rich phase protruding higher than the $A$-rich phase.

$g_{1}=0.18, h_{D}=g_{D}=0 \quad(B$-attracting substrate, neutral surface); and for "symmetric" films $h_{1}=h_{D}=-0.05, g_{1}=$ $g_{D}=0.18$ of Fig. 6 ( $B$-attracting substrate and surface). Random thermal currents $J^{T}$ from a Gaussian distribution of width 0.01 were applied in the diffusion step [providing an additional term $+\nabla \cdot\left(\Delta z J^{T}\right)$ to Eq. (6) $\left.[20,28]\right]$.

We use our model to investigate the evolution of an initially homogeneous film. Our results show that stages of roughening can be attributed to stages of phase separation (a quantitative discussion of how free energy, film roughness, and lateral phase separation evolve with time is given in the Supplemental Material [20]). Figures 2 and 4 show an antisymmetric and an asymmetric film, respectively, at different stages in evolution. In both cases, the film initially undergoes vertical stratification due to the preferential surface attraction, forming a bilayer. This bilayer then breaks up via the mechanism given in Ref. [27], which describes how the order parameter at the film surfaces divides as the film becomes laterally segregated (bilayer instability is also studied in [17] and [18]). Figures 3 and 5 are $2 \mathrm{D}$ plots showing the surface volume fraction (top, color matches 3D plots) and the film height (bottom, color bar shown) for the films in Figs. 2 and 4, respectively. Time increases from left to right, showing the breakup of the bilayer (left), the emergence of lateral phases (center), and the laterally segregated film (right) at the same time as the corresponding 3D plots.

When the film is in the bilayer state (Figs. 2 and 4 top) there is no significant roughening of the film surface. As the bilayer begins to break up (Figs. 3 and 5 left), showing significant phase separation at the top surface, the surface roughens quite significantly. This may correspond to the increased roughness of the surface prior to lateral phase separation of the bilayer, as reported in Ref. [6]. It seems that the onset of phase separation in the lateral direction induces this roughening. As the lateral phases emerge due
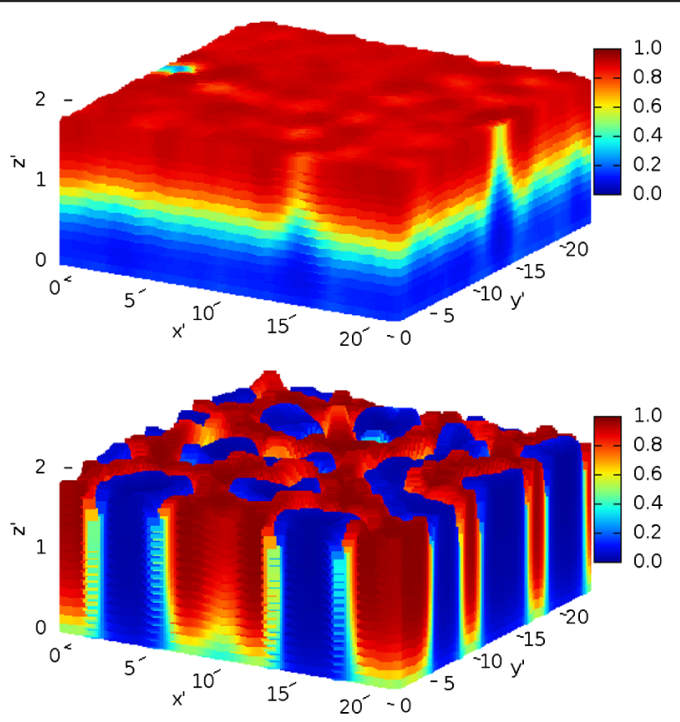

FIG. 4 (color online). Bilayer (top) at $\tau=160$ and laterally segregated state (bottom) at $\tau=1200$ for an asymmetric film of average height $h^{\prime}=1.73$. The $B$-rich phase breaks the surface even though the film is vertically stratified.

to the breakup of the bilayer (Figs. 3 and 5 center), dewetting couples strongly to the lateral phase separation, causing the surface roughening to shadow the phase separating morphology. Figure 5 (left) shows earlier signs of this coupling where the $B$-rich material has broken the surface.

The laterally segregated morphology that results depends on the surface regime. Figure 3 shows that the antisymmetric film laterally phase separates into a bicontinuous morphology of both the $A$-rich and $B$-rich phases. Our simulations show that the $B$-rich phase protrudes from the $A$-attracting film surface. From a purely thermodynamic perspective that considers the lateral phases in $1 \mathrm{D}$, it is not

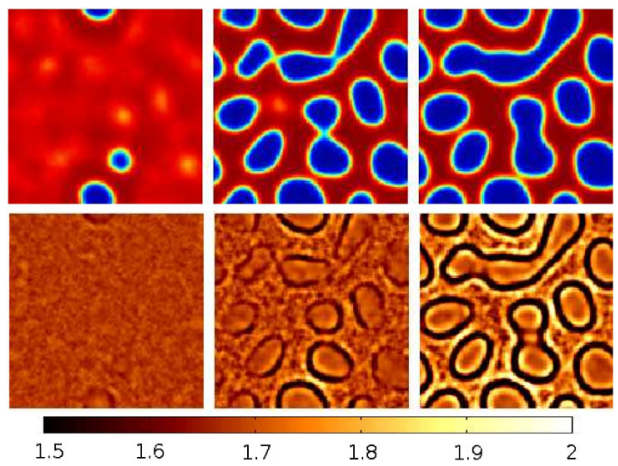

FIG. 5 (color online). Surface view for film shown in Fig. 4 (top: volume fraction, color bar of Fig. 4; bottom: film height, color bar shown). Left: beginning of breakup of the bilayer, $(\tau=400)$; center: more lateral phases break the surface, destroying the bilayer $(\tau=840)$; right: further roughening as lateral phases coarsen $(\tau=1200)$. The $A$-rich phase is continuous, encapsulating islands of the $B$-rich phase. 


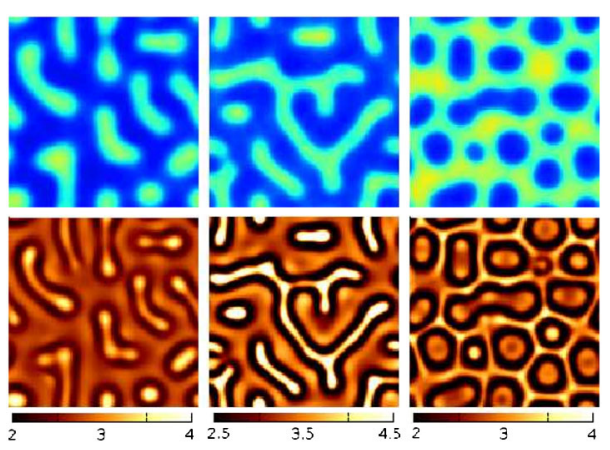

FIG. 6 (color online). Surface view of the laterally segregated states of three symmetric films, with different $\bar{\phi}$, which initially form a trilayer (top: volume fraction, as in Figs. 2 and 4; bottom: film height, color bar shown). Note that scaling depends on $\chi_{S}$, which varies with $\bar{\phi}$. Left: $\left(\sigma=0.03, \bar{\phi}=0.2, h^{\prime}=2.74\right)$ droplets of $A$-rich phase encapsulated by $B$-rich phase $(\tau=700)$; center: $\left(\sigma=0.01, \bar{\phi}=0.3, h^{\prime}=3.24\right)$ droplets join up on increasing $A$-component $(\tau=980)$; right: $(\sigma=0.01$, $\left.\bar{\phi}=0.5, h^{\prime}=2.60\right) A$-rich phase forms a protruding matrix encapsulating the $B$-rich phase $(\tau=1200)$.

clear why either phase would be higher than the other [29]. However, the kinetics of the breakup of the bilayer appears to promote the $B$-rich phase, which previously formed the bottom layer of the bilayer. The laterally segregated state of the asymmetric film shown in Fig. 5 is a continuous $A$-rich phase which encapsulates islands of the $B$-rich phase; the $B$-attracting substrate causes an excess of the $A$-component in the rest of the film.

For the asymmetric film of Fig. 4, an important point can be made about the bilayer (top): the film is definitely vertically stratified, even though the surface of the film would suggest lateral segregation (the snapshot has been chosen to show this). The lateral phase separation is proceeding from the top surface in this case [27,30]. The variations that can be seen in the bilayer correspond to where the lateral phases are in the laterally segregated film. In Ref. [31], the authors observed that a bicontinuous morphology is maintained throughout the spin-casting process, and conclude that this means no bilayer initially formed. Their conclusions therefore contradict earlier results on bilayer formation [6]. Our results bring these two contradicting interpretations together, showing that phase separation observed at the surface does not indicate that there is no vertical stratification.

We also performed simulations of symmetric films, in which vertical stratification produces a trilayer (an $A$-rich layer between two $B$-rich layers). We have only shown, in Fig. 6, the surface profiles of the final laterally segregated state, for various blend ratios (values of $\bar{\phi}$ ). For the symmetric blend $\bar{\phi}=0.5$, the final roughened morphology highly resembles that found in experiments, when the encapsulating phase protrudes from the film $[3,5]$. When the nonwetting $A$ component is the minority component, the protruding $A$-rich lateral phase becomes encapsulated in the $B$-rich phase. For $\bar{\phi}=0.2$, droplets of the minority phase form, which could form pancakelike droplets if other hydrodynamic flow mechanisms like those in Refs. [2,4] were present.

We have presented results from a new model for thin films of multicomponent fluids applied to binary polymer blend thin films. Our results show that roughening couples to phase separation, resulting in stages of surface roughening corresponding to distinct stages of phase separation. Our results can be interpreted in terms of phase equilibria [27,30]: the transient wetting layer that initially forms is a metastable state, the laterally segregated state containing the equilibria of global equilibrium. It seems to be energetically favorable for dewetting to occur at the interfaces between the laterally coexisting phases, so there is little route to lower the free energy of the film by roughening of the transient wetting layer, and roughening begins with the onset of lateral phase separation as a means to lower the free energy of the film as lateral inhomogeneities grow (see Supplemental Material for a quantitative discussion [20]).

[1] S. Walheim, M. Böltau, J. Mlynek, G. Krausch, and U. Steiner, Macromolecules 30, 4995 (1997).

[2] A. Karim, T. M. Slawecki, S. K. Kumar, J. F. Douglas, S. K. Satija, C. C. Han, T. P. Russell, Y. Liu, R. Overney, J. Sokolov, and M. H. Rafailovich, Macromolecules 31, 857 (1998).

[3] J. Heier, E. J. Kramer, P. Revesz, G. Battistig, and F. S. Bates, Macromolecules 32, 3758 (1999).

[4] H. Wang and R. J. Composto, Europhys. Lett. 50, 622 (2000).

[5] J. S. Kim, P. K. H. Ho, C. E. Murphy, and R. H. Friend, Macromolecules 37, 2861 (2004).

[6] S. Y. Heriot and R. A. L. Jones, Nat. Mater. 4, 782 (2005).

[7] A. D. F. Dunbar, P. Mokarian-Tabari, A. J. Parnell, S. J. Martin, M. W. A. Skoda, and R. A. L. Jones, Eur. Phys. J. E 31, 369 (2010).

[8] S. Ebbens, R. Hodgkinson, A. J. Parnell, A. D. F. Dunbar, S. J. Martin, P. D. Topham, N. Clarke, and J. R. Howse, ACS Nano 5, 5124 (2011).

[9] R. Craster and O. Matar, Rev. Mod. Phys. 81, 1131 (2009).

[10] M. Geoghegan and G. Krausch, Prog. Polym. Sci. 28, 261 (2003).

[11] N. Clarke, Eur. Phys. J. E 14, 207 (2004).

[12] N. Clarke, Macromolecules 38, 6775 (2005).

[13] K. R. Thomas, N. Clarke, R. Poetes, M. Morariu, and U. Steiner, Soft Matter 6, 3517 (2010).

[14] L. ONáraigh and J. L. Thiffeault, Phys. Rev. E 76, 035303 (2007).

[15] A. Pototsky, M. Bestehorn, D. Merkt, and U. Thiele, J. Chem. Phys. 122, 224711 (2005).

[16] A. Pototsky, M. Bestehorn, D. Merkt, and U. Thiele, Europhys. Lett. 74, 665 (2006).

[17] O. A. Frolovskaya, A. A. Nepomnyashchy, A. Oron, and A. A. Golovin, Phys. Fluids 20, 112105 (2008).

[18] S. Madruga and U. Thiele, Phys. Fluids 21, 062104 (2009). 
[19] H. Morita, T. Kawakatsu, and M. Doi, Macromolecules 34, 8777 (2001).

[20] See Supplemental Material at http://link.aps.org/ supplemental/10.1103/PhysRevLett.113.218301, which includes Refs. [21,22], for information regarding implementation of the model and a quantitative plot and discussion of how the free energy, film roughness, and degree of lateral phase separation evolve with time.

[21] I. C. Henderson and N. Clarke, Macromol. Theory Simul. 14, 435 (2005).

[22] J. I. Fukuda, M. Yoneya, and H. Yokoyama, Phys. Rev. E 73, 066706 (2006).

[23] P. G. de Gennes, J. Chem. Phys. 72, 4756 (1980).

[24] H. Nakanishi and P. Pincus, J. Chem. Phys. 79, 997 (1983).
[25] I. Schmidt and K. Binder, J. Phys. 46, 1631 (1985).

[26] U. Thiele, D. V. Todorova, and H. Lopez, Phys. Rev. Lett. 111, 117801 (2013).

[27] S. Coveney and N. Clarke, Phys. Rev. E 89, 062603 (2014).

[28] S. K. Das, S. Puri, J. Horbach, and K. Binder, Phys. Rev. E 72, 061603 (2005).

[29] S. Coveney and N. Clarke, J. Chem. Phys. 137, 174901 (2012).

[30] S. Coveney and N. Clarke, Phys. Rev. Lett. 111, 125702 (2013).

[31] D. T. W. Toolan, E. ul Haq, A. Dunbar, S. Ebbens, N. Clarke, P. D. Topham, and J. R. Howse, J. Polym. Sci. Part B Polym. Phys. 51, 875 (2013). 